\title{
Frequency of word use predicts rates of lexical evolution throughout Indo-European history
}

\author{
Mark Pagel, Quentin D. Atkinson and Andrew Meade
}

School of Biological Sciences, University of Reading, Whiteknights, Reading, Berkshire, RG6 6AS, UNITED KINGDOM

Greek speakers say 'ovpó', Germans 'schwanz', and the French 'queue' to describe what English speakers call a 'tail', but all of these languages use a related form of 'two' to describe the number after one. Among over one hundred IndoEuropean languages and dialects, the words for some meanings, such as 'tail', evolve rapidly, being expressed across languages by dozens of unrelated words, whilst others evolve much more slowly, such as the number 'two' for which all Indo-European language speakers use the same related word-form ${ }^{1,2}$. No general linguistic mechanism has been advanced to explain this striking variation in rates of lexical replacement among meanings. Here we use four large and divergent language corpora (English ${ }^{3}$, Spanish $^{4}$, Russian $^{5}$ and Greek ${ }^{6}$ ) and a comparative database of 200 fundamental vocabulary meanings in 87 Indo-European languages $^{2}$ to show that the frequency with which these words are used in modern language predicts their rate of replacement over thousands of years of IndoEuropean language evolution. Across all 200 meanings, frequently used words evolve at slower rates and infrequently used words evolve more rapidly. This relationship holds separately and identically across parts of speech for each of the four language corpora and accounts for approximately $50 \%$ of the variation observed in historical rates of lexical replacement. We propose that the frequency with which specific words are used in everyday language exerts a general and lawlike influence on their rates of evolution. Our findings are consistent with social models of word change that emphasise the role of selection, and suggest that owing 
to the ways that humans use language, some words will evolve slowly and others rapidly across all languages.

Languages, like species, evolve via a process of descent with modification (Table S1). The remarkable diversity of languages -- there are about 7,000 known living languages $^{7}$-- is a product of this process acting over thousands of years. Ancestral languages split to form daughter languages that slowly diverge as shared lexical, phonological and grammatical features are replaced by novel forms. In the study of lexical change, the basic unit of analysis is the cognate. Cognates are words of similar meaning with systematic sound correspondences indicating they are related by common ancestry. For example, cognates meaning "water" exist in English (water), German (wasser), Swedish (vatten) and Gothic (wato), reflecting descent from proto-Germanic (*water).

Early lexicostatistical ${ }^{8}$ studies of cognate diversity in Malayo-Polynesian and Indo-European language families revealed that rates of evolution vary across meaning categories $^{1,9}$. More recently we have obtained direct estimates of rates of cognate replacement on linguistic phylogenies (family trees) of Indo-European and Bantu languages, using a statistical model of word evolution in a bayesian Markov chain Monte Carlo (MCMC) framework ${ }^{10}$. We found that rates of cognate replacement varied among meanings and that rates for different meanings in Indo-European were correlated with their paired meanings in the Bantu languages. This indicates that variation in the rates of lexical replacement among meanings is not merely an historical accident, but rather is linked to some general process of language evolution.

Social and demographic factors proposed to affect rates of language change within populations of speakers, include social status ${ }^{11}$, the strength of social ties ${ }^{12}$, the size of the population ${ }^{13}$, and levels of outside contact ${ }^{14}$. These forces may influence rates of 
evolution on a local and temporally specific scale, however, they do not make general predictions across language families about differences in the rate of lexical replacement among meanings. Drawing on concepts from theories of molecular ${ }^{15}$ and cultural evolution $^{16,17,18}$, we suggest that the frequency with which different meanings are used in everyday language may affect the rate at which new words arise and become adopted in populations of speakers. If frequency of meaning use is a shared and stable feature of human languages, then this could provide a general mechanism to explain the large differences across meanings in observed rates of lexical replacement. Here we test this idea by examining the relationship between the rates at which Indo-European language speakers adopt new words for a given meaning and the frequency with which those meanings are used in everyday language.

We estimated the rates of lexical evolution for 200 fundamental vocabulary meanings $s^{8}$ in 87 Indo-European languages ${ }^{2,19}$. Rates were estimated using a statistical likelihood model of word evolution ${ }^{10}$ applied to phylogenetic trees of the 87 languages ${ }^{19}$ (Figure S1). The number of cognates observed per meaning varied from one to fortysix. For each of the 200 meanings we calculated the mean of the posterior distribution of rates as derived from a bayesian MCMC model that simultaneously accounts for uncertainty in the parameters of the model of cognate replacement and in the phylogenetic tree of the languages (see Methods). Rate estimates were scaled to represent the expected number of cognate replacements per 10,000 years, assuming a 8,700 -year age for the Indo-European language family ${ }^{19}$. Opinions on the age of IndoEuropean vary between approximately $6,000 \mathrm{BP}$ and $10,000 \mathrm{BP}^{20,21}$. Using a different calibration would change the absolute values of the rates but not their relative values.

Figure 1a shows the inferred distribution of rate estimates, where we observe a roughly 100-fold variation in rates of lexical evolution among the meanings. At the slow end of the distribution, the rates predict zero to one cognate replacements per 
Pagel, M., Atkinson, Q. D. and Meade, A. (2007). Frequency of word-use predicts rates of lexical evolution throughout IndoEuropean history. Nature, 449: 717-20.

10,000 years for words such as 'two', 'who', 'tongue', 'night', 'one' and 'to die'. By comparison, for the faster evolving words such as 'dirty', 'to turn', 'to stab' and 'guts' we predict up to nine cognate replacements in the same time period. In the historical context of the Indo-European language family this range yields an expectation of between $0-1$ and 43 lexical replacements throughout the $\sim 130,000$ language-years of evolution the linguistic tree represents, very close to the observed range in the fundamental vocabulary of 1 to 46 distinct cognate classes among the different meanings. These rates can be converted to estimates of the linguistic half-life ${ }^{10}$ (see Methods), or the time in which there is a $50 \%$ chance the word will be replaced by a different non-cognate form. These times vary from 750 years for the fastest evolving words to over 10,000 years for the slowest (Figure 1b).

We used spoken and written language corpus data from English ${ }^{3}$, Spanish $^{4}$, Russian $^{5}$ and Greek ${ }^{6}$ to measure the frequency of meaning use (Table S2). These languages sample from across the Indo-European language family (Figure S1) and their corpora were selected to provide large samples of language use (20-100 million words each). Figure 2 shows that the distribution of word use frequencies in each language is highly skewed such that most words are used relatively infrequently (less than 100 times per million words), with a small number of frequently used words (as often as 35,000 times per million words) accounting for most speech. Word use frequencies are highly correlated among the four languages $(0.78<r<0.89$, mean $r=0.84$; Figure S2) showing that words used at a high frequency in one language, tend to be used at a high frequency in the other languages. Because the four languages span the Indo-European tree, this suggests that frequency values are representative of Indo-European language use, and that frequencies of meaning use have been remarkably stable throughout IndoEuropean history (Figure S1). 
Figure 3 plots the rate of lexical replacement against frequency of word use for the 200 meanings. Separately in each corpus we observe a negative relationship (bold black line) between word frequency and rate (English, $r=-0.37$; Spanish, $r=-0.35$; Russian, $r=-0.41$; and Greek, $r=-0.32$ : all $p<0.0001)$. In all four languages, the more a meaning is used today, the slower its rate of evolution has been throughout the 6,000 - to 10,000-year history of Indo-European.

Some parts of speech are used more than others, so it is possible that the observed relationship arises from an effect of part of speech on rates of evolution. To examine this effect, we categorised meanings as either nouns, adjectives, verbs, pronouns, numbers, conjunctions, prepositions, or special adverbs ('what', 'when', 'where', 'how', 'here', 'there' and 'not'). We then predicted variation in rates of lexical replacement from a regression model allowing both of these effects to operate simultaneously, thereby controlling for one another. The inverse relationship between frequency of meaning use and rate of lexical evolution holds separately for parts of speech (Figure 3 coloured lines). For a given frequency of meaning use, prepositions and conjunctions evolve most quickly, followed by progressively slower evolution for adjectives, verbs, nouns, special adverbs, pronouns and finally numbers. The rank order of effects for part of speech is identical across the four corpora, and the combined models account for approximately $50 \%$ of the variance in rates of lexical evolution (English, $R=0.69$; Spanish, $R=0.69$; Russian, $R=0.71$; and Greek, $R=0.69$ : all $p<0.0001)$. Adding an interaction effect between part of speech and frequency of meaning use did not improve the fit of the model: frequency of meaning use affects the rate of evolution in the same way for each part of speech.

The consistent pattern across the four languages in the relative rates of lexical replacement among parts of speech may help us to understand the mechanism by which word-use frequency affects rate of evolution. Frequency of word use could directly 
modify the rate at which new word forms arise, with fewer spontaneous errors occurring for highly expressed words. Errors in word perception, recall and production have been shown to decrease with word frequency according to the 'power law of learning, ${ }^{22,23}$. Alternatively, the rate at which new forms appear could be the same for all meanings, with frequency of use affecting the probability that a population of speakers will come to adopt a given innovation. This suggests some form of linguistic, frequencydependent, purifying selection is responsible for the slow rate of evolution of highly expressed words. Innovations, being rare, may not be favoured in speech because there is an increased chance that they will be misinterpreted. This effect should be stronger the more often the meaning is required in speech or the more important it is to the meaning of speech.

In each of our language corpora, numbers, pronouns and the special adverbs evolve the most slowly for a given frequency of word use. These parts of speech seem important to the meaning of spoken communication, and may therefore be subject to stronger selection. The rapidly evolving parts of speech include conjunctions, prepositions and adjectives whose exact forms may be less important to conveying meaning. There is also evidence in natural populations of speakers that when more than one word is used to express the same meaning, the relative frequencies of use of the rare words is lower than expected from a neutral drift model of evolution ${ }^{24}$, consistent with selection against innovations. These findings may then indicate that the purifying selection model of word evolution provides a more accurate description of how words evolve within populations of speakers. It is also consistent with models of cultural and linguistic evolution that incorporate a conformist bias ${ }^{16,17}$, although these models cannot identify a priori which words will be subjected most strongly to such effects.

Our findings, based on a sample of fundamental vocabulary items, identify a general mechanism of linguistic evolution, which is expected to operate similarly across 
all languages and time scales and makes predictions about rates associated with specific meanings. To the extent that the structure and everyday functions of human verbal communication mean that some words will tend to be used more frequently in all languages, we expect these words to evolve slowly, and vice versa for infrequently used words. Combined with parts of speech this simple factor allows us to account for about $50 \%$ of the variance in rates of lexical replacement throughout the 6,000 - to 10,000 -year history of Indo-European languages. The generality of this influence is suggested in the finding that estimates of the rate of lexical replacement in Indo-European languages are correlated with rate estimates in Bantu ${ }^{10}$, Cushitic and Malayo-Polynesian ${ }^{1}$.

Being able to link variation in rates of lexical replacement to the frequency of word use also provides insights into some features of comparative linguistics. One is that we expect languages to diverge initially in the least frequently used parts of their vocabularies. This may mean that languages retain mutual intelligibility far longer than expected from simple uniform rates models of linguistic divergence ${ }^{8}$. Related to this, the words for frequently used meanings should, on average, be less prone to borrowing during language contact. Higher frequency words may also be more likely to exhibit ancestral morphology. Irregular verbs in English often retain their ancestral morphology, and are among the most frequently expressed verbs ${ }^{25}$. Finally, we note that our rate estimates show that some words evolve slowly enough to allow homologous lexical forms to persist for tens of thousands of years. These slow rates demonstrate that humans are capable of producing a culturally transmitted replicator, that perhaps because of the purifying force of spoken word frequency, can have a replication accuracy as high as that of some genes ${ }^{26}$. Along with continued efforts at identifying cognate words separated by thousands of years of sound change ${ }^{27}$, this raises the possibility of using selected lexical items to evaluate hypothesized 'long range' linguistic relationships such as Eurasiatic ${ }^{28}$ and Nostratic ${ }^{29}$. 


\section{Methods Summary}

Cognate data We grouped the words for each of the 200 meanings in the Swadesh fundamental vocabulary ${ }^{8}$ into cognate sets using the classifications in the comparative Indo-European database ${ }^{2}$. Meanings had between 1 and 46 cognate sets across the 87 languages in our study, producing a total of 4,049 cognates (including unique wordforms).

Phylogenetic trees We inferred a bayesian posterior distribution of phylogenetic trees of the 87 languages from a binary data matrix derived from the cognacy classifications. The 4049 binary vectors in the matrix code for the presence (' 1 ') or absence (' 0 ') of each of the 4,049 cognates ${ }^{2,30}$. The consensus tree of this posterior sample is reported in Figure S1.

Rates of lexical replacement We categorised words for each of the 200 meanings into $k$ states representing the $k$ different cognate classes identified for that meaning. For example, $\mathrm{k}=24$ for the meaning class 'big' because it has 24 cognates among the 87 languages. For each meaning, we estimate the instantaneous transition rate, $q$, from any state (cognate class) $i$ to any state $j$, as the mean of its bayesian posterior distribution of rates, summed over models of evolution and phylogenetic trees in the posterior sample of trees. This accounts for uncertainty in the model of evolution and in the phylogenetic tree, and does not suffer from loss of information owing to the conversion of cognate data to pair-wise similarity scores between languages. Half-life estimates were derived as described in reference 10 .

Word frequency data We obtained word use frequencies from English ${ }^{3}, \mathrm{Spanish}^{4}$, Russian $^{5}$ and Greek ${ }^{6}$ corpora, combining the frequencies for all words comprising a shared canonical form (e.g., 'push', 'pushes', 'pushing', and 'pushed'). 
Pagel, M., Atkinson, Q. D. and Meade, A. (2007). Frequency of word-use predicts rates of lexical evolution throughout IndoEuropean history. Nature, 449: 717-20.

\section{References}

1. Kruskal, J. B., Dyen, I., \& Black, P. D. in Mathematics in the archaeological and historical sciences (eds Hodson, F. R., Kendall, D. G. \& Tautu, P.) 361-380 (Edinburgh University Press, Edinburgh, 1971).

2. Dyen, I., Kruskal, J. B. \& Black, P. An Indo-European Classification, a Lexicostatistical Experiment .1 Transactions of the American Philosophical Society 82, 1-132 (1992).

3. Leech, G., Rayson, P. \& Wilson, A. Word Frequencies in Written and Spoken English: based on the British National Corpus (Longman, London, 2001).

4. Davies, M. Corpus del Español (http://www.corpusdelespanol.org). 100 million words, 1200s-1900s. 2001-2002.

5. Sharoff, S. in Corpus Linguistics Around the World (eds Archer, D., Wilson, A. \& Rayson, P.) 167-180 (Rodopi, Amsterdam, 2005).

6. Hellenic National Corpus ${ }^{\mathrm{TM}}$ (HNC) Web Version 3.0 Copyright (C) 1999-2006 ILSP (http://hnc.ilsp.gr/).

7. Gordon, Raymond G., Jr. (ed.), Ethnologue: Languages of the World, Fifteenth edition. Dallas, Tex.: SIL International. Online version: http://www.ethnologue.com/ (2005).

8. Swadesh, M. Lexico-statistic dating of prehistoric ethnic contacts. Proceedings of the American Philosophical Society 96, 453-63 (1952).

9. Dyen, I., James, A. T. \& Cole, J. W. L. Language divergence and estimated word retention rate. Language 43, 150-171 (1967).

10. Pagel, M. \& Meade, A. in Phylogenetic methods and the prehistory of languages. (eds Clackson, J., Forster P., and Renfrew, C.) 173-182 (McDonald Institute for Archaeological Research, Cambridge, UK, 2006). 
Pagel, M., Atkinson, Q. D. and Meade, A. (2007). Frequency of word-use predicts rates of lexical evolution throughout IndoEuropean history. Nature, 449: 717-20.

11. Labov, W. Principles of Language Change. Volume 2: Social Factors (Blackwell, Malden, MA, 2001)

12. Milroy, J. \& Milroy, L. Linguistic Change, Social Network and Speaker Innovation. Journal of Linguistics 21, 229-284 (1985).

13. Nettle, D. Is the rate of linguistic change constant? Lingua 108, 119-136 (1999).

14. Thomason, S. \& Kaufman, T. Language contact creolization, and genetic linguistics (University of California Press, Berkeley, CA, 1988).

15. Kimura, M. The neutral theory of molecular evolution (Cambridge University Press, Cambridge, 1983).

16. Boyd, R. \& Richerson, P. J. Culture and the Evolutionary Process. (University of Chicago Press, Chicago, 1985).

17. Kirby, S. Function, selection, and innateness - the emergence of language universals (Oxford University Press, Oxford, 1999).

18. Croft, W. Explaining language change: An evolutionary approach. (Pearson Education Ltd., Harlow, 2000).

19. Gray, R. D. \& Atkinson, Q. D. Language-tree divergence times support the Anatolian theory of Indo-European origin. Nature 426, 435-439 (2003).

20. Gimbutas, M. Old Europe c. 7000-3500 B.C., the earliest European cultures before the infiltration of the Indo-European peoples. Journal of Indo-European Studies 1, 1-20 (1973).

21. Renfrew, C. Archaeology and Language: The Puzzle of Indo-European Origins (Cape, London, 1987).

22. Anderson, J. R. Acquisition of cognitive skill. Psychology Review 89, 369-406 (1982). 
23. Ellis, N. C. Frequency effects in language processing: A review with implications for theories of implicit and explicit language acquisition. Studies in Second Language Acquisition 24, 143-188 (2002).

24. Fontanari, J. F. \& Perlovsky, L. I. Solvable null model for the distribution of word frequencies. Physical Review E 70, 042901 (2004).

25. Francis, N. \& Kucera, H. Frequency Analysis of English Usage: Lexicon and Grammar (Houghton Mifflin, New York, 1982).

26. Burger, J. et al. Absence of the lactase-persistence-associated allele in early Neolithic Europeans. Proc Natl Acad Sci US A 104, 3736-3741 (2007).

27. Mackay, W. \& Kondrak, G. Comparing word similarity and identifying cognates with pair hidden Markov models. Proceedings of the 9th Conference on Natural Language Learning (CoNLL), 40-47 (ACL, Shroudsburg, Penn., 2005).

28. Greenberg, J. H. Indo-European and Its Closest Relatives: The Eurasiatic Language Family. Volume 1, Grammar. (Stanford University Press, Stanford, CA, 2000).

29. Kaiser, M. \& Shevoroshkin, V. Nostratic. Annual Review of Anthropology 17, 309-330 (1988).

30. Pagel, M., and Meade, A. A phylogenetic mixture model for detecting patternheterogeneity in gene sequence or character-state data. Syst. Biol. 53, 571-81 (2004). 


\section{Methods Section}

Cognate Data We used the comparative Indo-European database ${ }^{2}$, which records word forms and cognacy judgements in 95 languages across the 200 terms in the Swadesh fundamental vocabulary ${ }^{8}$. We excluded 11 of the speech varieties that had not been coded by Dyen $e t a{ }^{2}$ and were identified by them as less reliable, leaving a sample of 84 languages. We added cognacy judgements for the same 200 meanings for three extinct Indo-European languages (Hittite, Tocharian A and Tocharian B), based on multiple sources ${ }^{19}$, for a combined sample of 87 languages. Meanings had from between 1 and 46 cognate sets across the 87 languages, for a total of 4,049 cognates, including unique words.

Phylogenetic Trees We inferred the posterior distribution of phylogenetic trees for the 87 languages using the Markov chain Monte Carlo (MCMC) methods ${ }^{31}$ implemented in BayesPhylogenies $^{30,32}$. The cognacy data were transformed to a binary matrix with rows representing the 87 languages and columns identifying the presence (' 1 ') or absence (' 0 ') of each of the 4,049 cognates (including unique 'cognates'). We then characterised the probability of these data on phylogenetic trees, using a two-state (presence/absence) continuous-time Markov transition rate mode ${ }^{10}$. The probability of the data $D$ given the model of evolution $M$ and a tree $T$ is written in the usual way as $P(D \mid M, T)=\prod_{c} P\left(D \mid \mathbf{Q}_{b}, T\right)$ where here the model of evolution for the binary vectors $\mathbf{Q}_{b}$ is the $2 \times 2$ matrix recording the rates of transition between the binary elements corresponding to the gain of the cognate class and the loss of the cognate class, and the product is over the $c=4049$ binary vectors that identify cognate classes. The elements of $\mathbf{Q}_{b}$ are given by $q \pi_{j}$ where $j$ represents the state (presence/absence) to which the cognate is moving and $\pi$ is the equilibrium frequency of the $j$ th state ${ }^{10,33}$. We used a single rate parameter $q$ and estimated the equilibrium frequencies of presence and absence from the data as part of the Markov chain. Transition rates were allowed to 
vary among cognate class vectors (successive vectors of the binary matrix) according to a gamma distribution ${ }^{34}$ with four rate categories. The shape parameter of the gamma distribution was estimated from the data.

We derived the posterior sample of trees from a Markov chain allowed to run for 40,000,000 generations. After discarding the first 2,500,000 generations as burn-in, we sampled every 50,000th tree in the chain to ensure that successive trees were statistically independent. This produced a posterior sample of 750 trees. Examination of autocorrelation times of the MCMC plots indicated runs had converged to the equilibrium distribution and showed very low autocorrelation, yielding an effective sample size of at least 500. The consensus tree of this posterior sample is reported in Figure S1.

Rates of lexical replacement We categorised the words for each of the 200 meanings into $k$ states representing the $k$ different cognate classes identified for that meaning. In the meaning class 'big', for example, $k=24$ because this meaning is represented by 24 cognates among the 87 languages. The probability of observing the distribution of the $k$ lexical terms for a meaning $m$ on any given tree can be written as $P\left(m \mid \mathbf{Q}_{m}, T\right)$ where $\mathbf{Q}_{m}$ is a $k \times k$ matrix of the transition rates from any cognate class $i$ to any other class $j$ for a particular meaning, and $T$ is the phylogenetic tree ${ }^{10}$. The elements of $\mathbf{Q}_{m}$ are given by $q \pi_{j}$ where $q$ is the instantaneous transition rate (as above but now for a particular meaning) and $\pi_{j}$ is the equilibrium frequency of state $j^{10}$. We estimate $\mathbf{Q}_{m}$ using a continuous-time Markov transition rate model ${ }^{10,29,33}$. The equilibrium frequencies are not known and therefore must either be estimated from the data or fixed at prior values. For the results reported in Figures 1 and 3, we assumed uniform equilibrium frequencies across cognates. Thus for a meaning represented by $k=3$ cognates, each $\pi_{j}$ is set to $1 / 3$. Because of the large number of cognate classes for some meanings ( $k=46$ for the meaning 'dirty') it is impractical to estimate $\pi_{j}$ from the 
Pagel, M., Atkinson, Q. D. and Meade, A. (2007). Frequency of word-use predicts rates of lexical evolution throughout IndoEuropean history. Nature, 449: 717-20.

data. However, using the observed empirical frequencies of the $k$ classes for each meaning across the 87 languages gives the same qualitative results reported in Figure 3 and none of our conclusions is altered.

The posterior distribution of the rate parameter $q$ is estimated from a Markov chain that simultaneously proposes new values for $q$ and samples new trees from the posterior distribution of $T$. We used the mean of the posterior distribution of $q$ to estimate the rates of lexical replacement reported in Figures 1 and 3. Our approach accounts for uncertainty in the model of evolution and in the phylogenetic tree, and, unlike earlier, lexicostatistical approaches to estimating rates of cognate replacement, does not suffer from information loss owing to the conversion of cognate data to pair-wise similarity scores between languages ${ }^{35}$. Mean and variance for the 200 meaning category rate estimates are provided in Table S2. Rate estimates are not expected to be biased across parts of speech by the process of grammaticalization (see Supplementary Information for details).

Half-life estimates were calculated from the mean $q$ values by solving $p=e^{-q t}$ for $t$, setting $p=0.5$ as described in reference 10 .

Word frequency data Word use frequencies were obtained for English, Spanish, Russian and Greek from the corpora databases described in references 3-6. Word frequencies were compiled by searching for all forms listed under the canonical form (or lemma) of each meaning. For example, for the verb meaning 'push' in English, we include 'push', 'pushes', 'pushing', and 'pushed'. Word frequency and part of speech data are provided in Table $\mathrm{S} 2$.

31. Metropolis, N., Rosenbluth, A. W., Rosenbluth, M. N., Teller, A. H., \& Teller, E. Equations of state calculations by fast computing machines. Journal of Chemical Physics 21, 1087-1091 (1953). 
Pagel, M., Atkinson, Q. D. and Meade, A. (2007). Frequency of word-use predicts rates of lexical evolution throughout IndoEuropean history. Nature, 449: 717-20.

32. Pagel, M. \& Meade, A. in Mathematics of evolution and phylogeny (ed. Gascuel, O.) 121-139 (Oxford Univiversty press, New York, 2005).

33. Pagel, M. \& Meade, A. In The evolution of cultural diversity: a phylogenetic approach (eds Mace, R., Holden, C., and Shennan, S.) 235-256 (UCL Press, London, 2005).

34. Yang, Z. Maximum likelihood phylogenetic estimation from DNA sequences with variable rates over sites: approximate methods. J Mol Evol 39, 306-314 (1994).

35. Steel, M., Hendy, M. \& Penny, D. Loss of information in genetic distances. Nature 333, 494-495 (1988).

Supplementary Information accompanies the paper on www.nature.com/nature.

Acknowledgements We thank Russell Gray and Simon Greenhill for useful comments and advice. This research is supported by a grant to MP from the Leverhulme Trust.

Competing Interests statement The authors declare that they have no competing financial interests.

Correspondence and requests for materials should be addressed to M.P. (e-mail: m.pagel@reading.ac.uk). 
Figure 1 | Frequency plots for rates of lexical evolution in Indo-European across 200 fundamental vocabulary meanings - a records the mean estimated rate of cognate replacement from the MCMC analysis for each meaning. $\mathbf{b}$ shows the same rate distribution converted to word half-lives ${ }^{10}$, or the time in which there is a $50 \%$ chance the word will be replaced by a different non-cognate form. The longest half-lives $(76,530$ years) are for meanings that show no change across Indo-European (see Supplementary Information).

Figure 2 | Distribution of frequency of word use for $\mathbf{2 0 0}$ meanings in four Indo-European languages - a English ${ }^{3}, \mathbf{b}$ Spanish $^{4}, \mathbf{c}$ Russian $^{5}$, and d Greek $^{6}$. These four languages sample from across the Indo-European language family. Figure S1 shows where each language fits on the Indo-European phylogeny. Word use frequencies are highly correlated among the four languages $(0.78<r<0.89$, mean $r=0.84$; see Figure $S 2)$.

Figure 3 | Log-log plots of frequency of meaning use against estimated rate of lexical evolution for $\mathbf{2 0 0}$ basic meanings in four Indo-European languages - a English, b Spanish, c Russian, and d Greek. A linear regression (bold black line) reveals a consistent negative relationship between the logfrequency of meaning use and log-rate of lexical replacement across all four languages (English, $r=0.37$; Spanish, $r=0.35$; Russian, $r=0.41$; and Greek, $r=0.32])$. Points are colour coded according to part of speech. Coloured lines show the results of a multiple regression including frequency and part of speech. The relationships are all consistently negative (English, $R=0.69$; Spanish, $R=0.69$; Russian, $R=0.71$; and Greek, $R=0.69)$. The height of each line in the four graphs indicates the relative speed of lexical evolution for a given frequency of meaning use for each part of speech. Conjunctions (grey) evolve fastest, followed by prepositions (turquoise), adjectives (red), verbs (blue), 
Pagel, M., Atkinson, Q. D. and Meade, A. (2007). Frequency of word-use predicts rates of lexical evolution throughout IndoEuropean history. Nature, 449: 717-20.

nouns (green), special adverbs (yellow), pronouns (orange) and numbers (purple). 
a

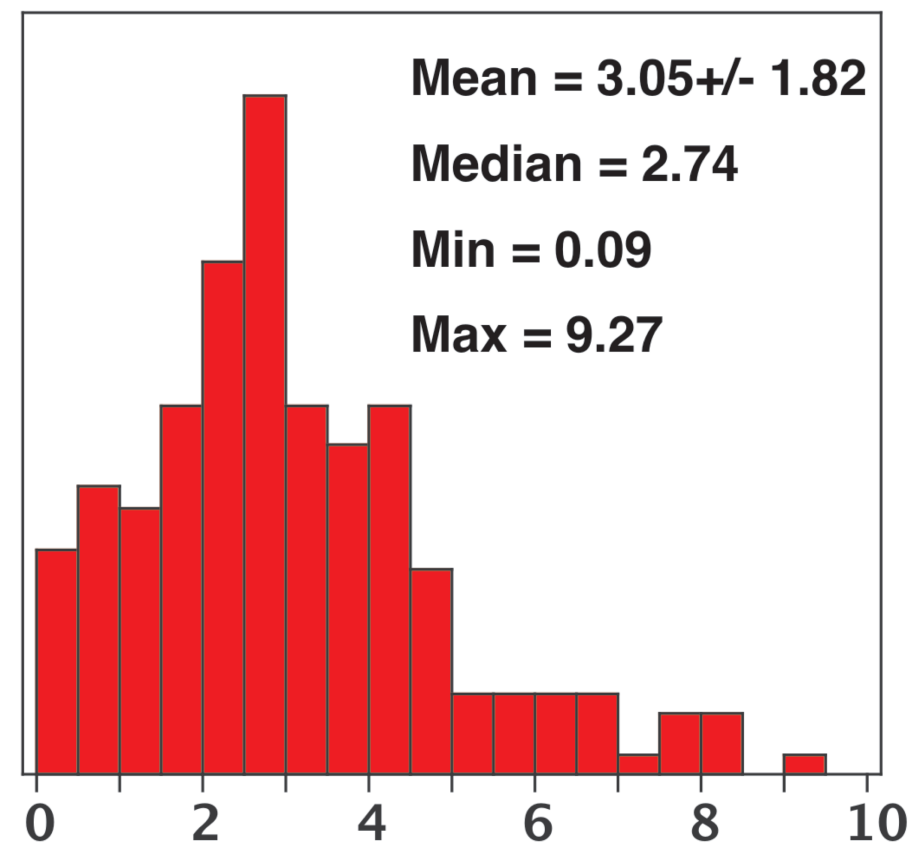

Mean word replacement rate per $10 \mathrm{k}$ years b

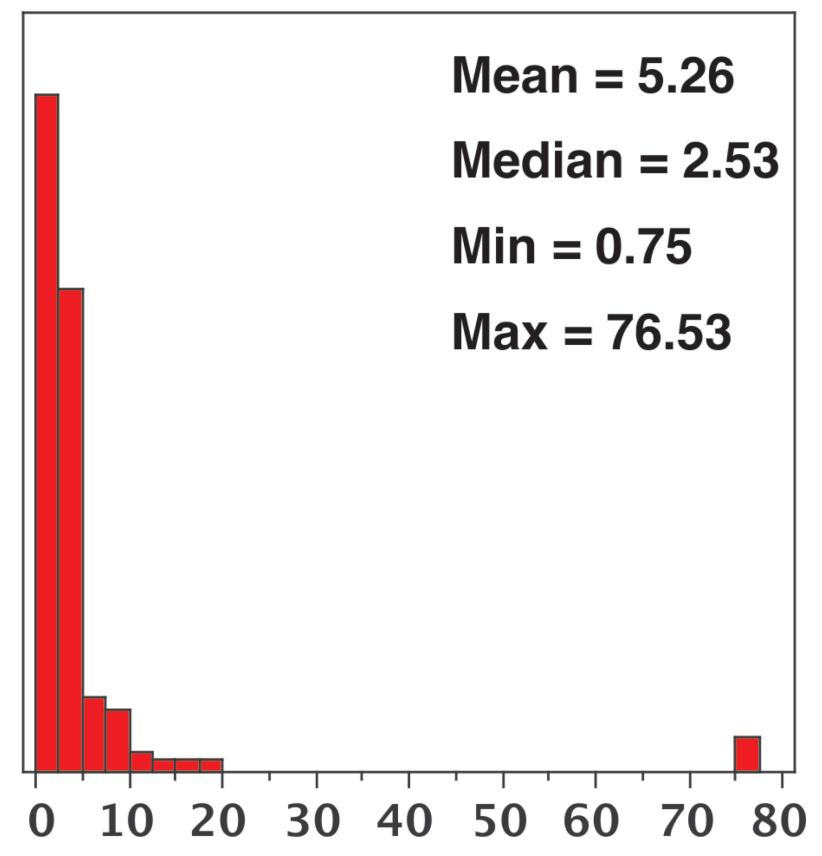

Mean word half-life in millenia 

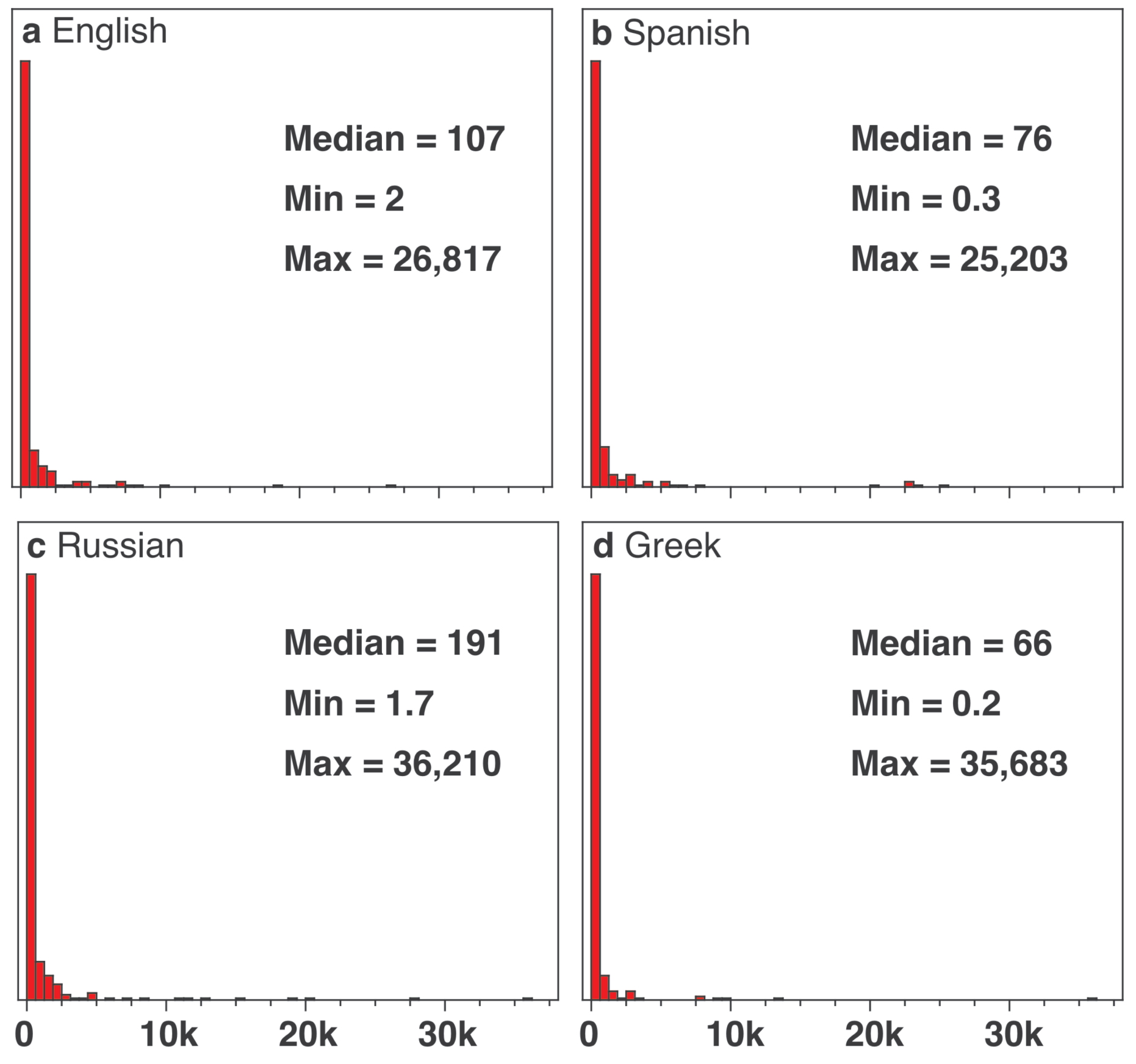

Frequency of word use per million words 

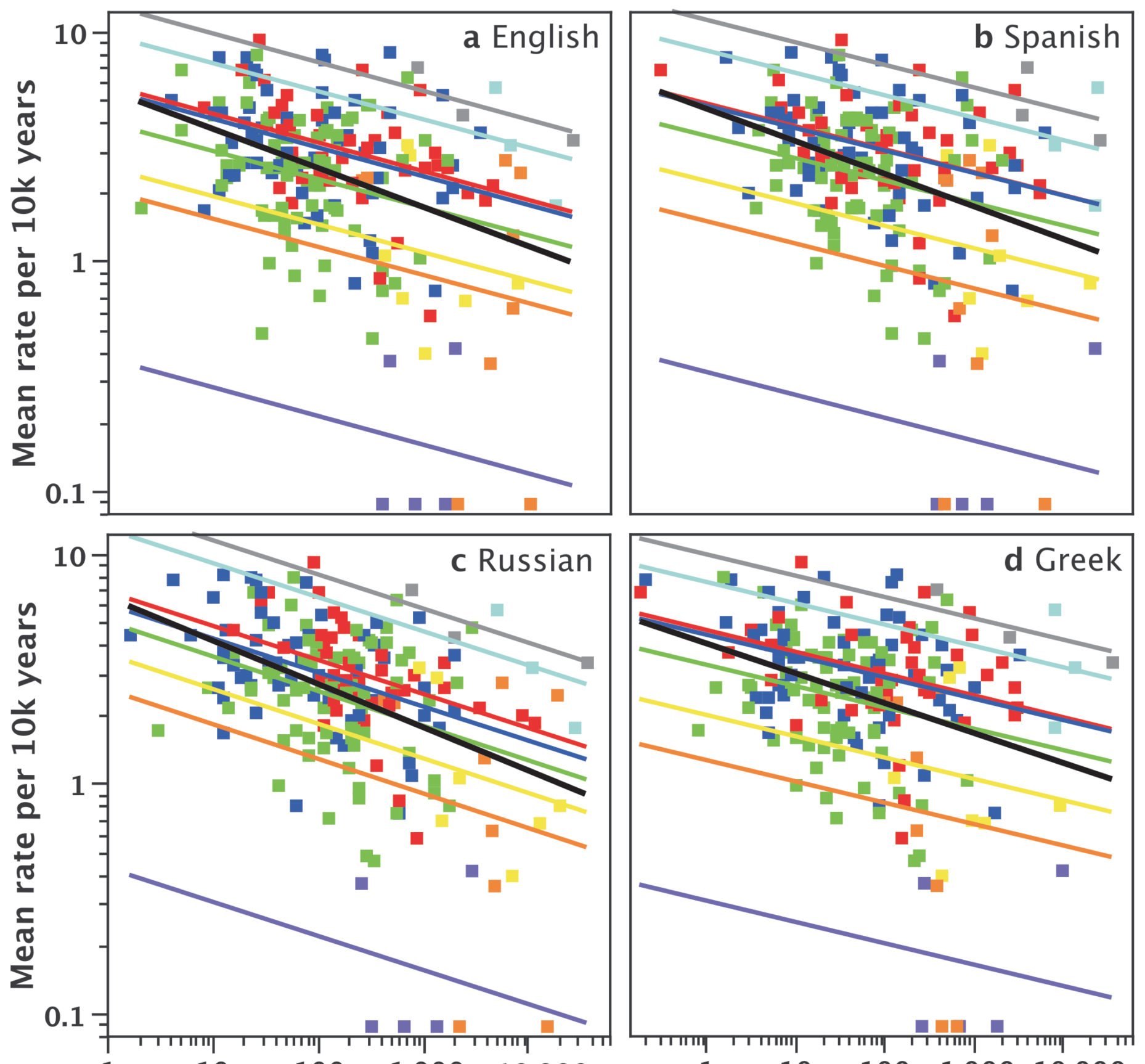

$\begin{array}{lllll}1 & 10 & 100 & 1,000 & 10,000\end{array}$

1

$\begin{array}{llll}10 & 100 & 1,000 & 10,000\end{array}$

Frequency of word use per million words 